Journal of Scientific Perspectives

Volume 3, Issue 3, Year 2019, pp. 215-222

E - ISSN: 2587-3008

URL: http://ratingacademy.com.tr/ojs/index.php/jsp

DOI: https://doi.org/10.26900/jsp.3.022

Research Article

\title{
EFFECTIVENESS OF NON-PHARMACOLOGICAL COMMUNITY - BASED NURSING INTERVENTIONS FOR SMOKING CESSATION IN ADULTS: STUDY PROTOCOL OF A SYSTEMATIC REVIEW
}

\author{
Handan TERZI * \& Yeter KİTiŞ ** \& Belgin AKIN *** \\ * Research Assistant, MSN, PhD., Candidate., Kastamonu University, Faculty of Health Sciences \\ Nursing Department TURKEY, e-mail: handanterzi@kastamonu.edu.tr \\ ORCID: https://orcid.org/0000-0001-8450-4481 \\ ** Assoc. Prof. Dr., PhD, MSN., Gazi University, Faculty of Health Sciences Nursing Department, \\ TURKEY,e-mail:ykitis@yahoo.com \\ ORCID: https://orcid.org/0000-0002-9246-8131 \\ *** Prof. Dr., PhD, MSN., Selçuk University, Nursing Faculty Public Health Nursing Department, \\ TURKEY, e-mail: akin.belgin@gmail.com \\ ORCID: https://orcid.org/0000-0002-8094-4110
}

Received: 10 July 2018; Accepted: 19 July 2019

\begin{abstract}
Tobacco use is one of the common and preventable risk factors of non-communicable diseases. Although the smoking rates tends to be decreased thanks to universal policies, it still is not satisfactory, especially among the middle-aged individuals. In order to achieve success in smoking cessation activities, it is crucial to determine the most effective nursing interventions and apply them into the practice. The objective of this systematic review is to investigate the effectiveness of nonpharmacological community-based nursing interventions for smoking cessation on cessation rate, nonrelapsing rate, knowledge belief and attitude related to smoking in adults living in the community. PubMed, Cochrane Library, EMBASE, PsycINFO, Cochrane Central Register of Controlled Trials (CENTRAL), EBSCO, ProQuest, Academic Search Complete, Ovid, CINAHL Plus with Full Text, MEDLINE, Web of Science, Scopus, Turkish Council of Higher Education Thesis Center, Ulakbim Turkish Medical Database and Turkiye Citation Index were searched. Databases were searched from the 1st of January 2008 to the 31st of December 2017. Languages were Turkish and English. The protocol of this systematic review was registered to PROSPERO database. The registration number is CRD42018088007. The study is ongoing. The databases were searched and 796 studies were retrieved in total. Data extraction process is ongoing. The data to be gathered from this systematic review is predicted to be a source for both primary care nurses and the other primary care professionals in terms of the effective smoking cessation method, its duration and changes in knowledge belief and attitude of individuals. The smoking cessation interventions were performed actively by primary care nurses, will facilitate to reach both local and global targets for preventing the non-communicable diseases. It is also aimed to contribute to nursing education curriculum by revealing the roles and influence of primary care nurses on smoking cessation promotion.
\end{abstract}

Keywords: adult; community health nursing; smoking cessation; primary care. 


\section{INTRODUCTION}

Tobacco use is one of the most common and preventable risk factors of noncommunicable diseases (WHO, 2013). Cigarette is known as the most popular tobacco products among adults (Jamal et al., 2018). Although the global prevention strategies in order to decrease the usage of tobacco and tobacco products have been effective so far, the prevalence of smoking in adults is still high (WHO, 2018). The global prevalence of tobacco use of the 15+ year-old individuals was reported as 20.2\%, in 2015 (WHO, 2018). In the WHO European Region, the prevalence was decreased by $4.6 \%$ (34.5\% to 29.9\%) between 2005-2015 (WHO, 2018). In Turkey, the prevalence of tobacco use of the $15+$ year-old individuals was stated as $27.3 \%$ in 2014 and $26.5 \%$ in 2016 (TUIK, 2017). Early adulthood (25-44 years old), male gender, lower income and education level were found to be associated with higher smoking prevalence in the literature (Asma et al., 2015; Jamal et al., 2018; WHO, 2018). In the European region, the rate of male tobacco use was higher than that of women (38.7\% and $21 \%$ respectively) (WHO, 2018). Likewise, the rate of male tobacco use $(40.1 \%)$ was approximately three times higher than that of women (13.3\%) in Turkey (TUIK, 2017).

Although the prevalence tends to be decreased it is still not satisfactory, especially among the middle-aged individuals (WHO, 2018). It was reported that the highest smoking prevalence among the 45+ age group was in India and Bangladesh, in 2015. Approximately $29 \%$ of 45-65 year-old individuals and 10\% of 65+ year elders are reported as current smokers in Turkey (Asma et al., 2015). Quitting smoking at any age has considerable impact on health status and life expectancy of individuals. In a global study, while the life expectancy of life time smokers was found to be decreased approximately 10 years, the life expectancy of individuals who stopped smoking at the ages of 40-50 or 60 was found to be increased nine-six and three years, respectively (Asma et al., 2015). The determination of the most effective smoking cessation interventions in the middle and advanced age periods is needed. It has been aimed to increase the success of smoking cessation prevalence, preventing tobacco dependence and relapses in the policies, adopted by Turkish Ministry of Health, along with the global tobacco control initiatives. According to this objective, educating primary health care providers related to smoking cessation intervention issue, monitoring smoking cessation services and widespread those in the community via technology have been targeted as essential strategies (TCSaglikBakanligi, 2015).

In order to achieve success in smoking cessation activities, it is crucial to determine the most effective interventions and apply them to the practice. In the literature, frequently used smoking cessation interventions were identified in three main topics as pharmacological, nonpharmacological and multi-modal approaches (Chen \& Wu, 2015). Non-pharmacological approaches, such as cognitive behavioral therapy, motivational support, enhancing selfefficacy, counseling and specifically structured educational interventions, were proven as more effective strategies in the current literature related to smoking cessation (Byers et al., 2018; Elshatarat, Yacoub, Khraim, Saleh, \& Afaneh, 2016; Hughes \& Naud, 2016; Moura, Menezes, Mariano, Silva, \& Sousa, 2011). In a meta-analysis, non-pharmacological and multi-modal approaches were found more effective than pharmacological treatment in smoking cessation in adults (Chen \& $\mathrm{Wu}, 2015)$. The main philosophy of health promotion is to teach healthy lifestyle behaviors to individuals and to support them maintaining these behaviors. In this context individuals should be empowered to take health self-responsibility, particularly via improving the effectiveness of primary health care services in smoking cessation (Elshatarat et al., 2016; Nichter, Carkoglu, Nichter, Ozcan, \& Uysal, 2018). Self-efficacy is known as a remarkable feature for smoking cessation. Verbal persuasion performed by health care providers for individuals who quitted smoking was stated to have a positive impact on their self-efficacy. The higher self-efficacy level was identified as the more successful the smoking 
cessation interventions and the more helpful for preventing the relapses (Elshatarat et al., 2016). In line with the literature, it can be said that especially the primary health care providers should role actively in smoking cessation strategies.

Nurses, the most crowded health care professionals across the globe, are known to have remarkable roles on smoking cessation issue both in the preventive services and in the hospital settings (Byers et al., 2018; Nichter et al., 2018; Sarna, Bialous, Chan, Hollen, \& O'Connell, 2013). It was stated in the literature that, although it was not statistically significant, nurse-led smoking cessation interventions were as effective as physician-led interventions in the primary care settings (Byers et al., 2018). In a meta-analysis, the smoking cessation interventions performed by nurses at the hospital settings were found to be more effective than those at the primary care services (Rice, Hartmann-Boyce, \& Stead, 2013). In the update of the same metaanalysis, the researchers stated that nurse-led smoking cessation interventions performed at both primary care and hospital settings increased the cessation success of the current smokers (Rice, Heath, Livingstone-Banks, \& Hartmann-Boyce, 2017). However, improvement of preventive health care behaviors when individuals faced with health problems is not an efficient approach, according to the contemporary preventive health care (Byers et al., 2018; Rice et al., 2017). It is also a cost-effective approach that providing preventive healthcare services both in the first appointment points of those services and in the places of individuals own.

There were numerous studies evaluated the effectiveness of smoking cessation interventions in the literature (Chen \& Wu, 2015; Rice et al., 2013; Rice et al., 2017; Zbikowski, Magnusson, Pockey, Tindle, \& Weaver, 2012). Nevertheless, there have no systematic reviews specifically indicate the effectiveness of smoking cessation interventions performed by nurses in the primary care settings. It is thought that nurse-performed smoking cessation interventions in the primary care settings will facilitate to reach the targets both at local and global levels for preventing the non-communicable diseases. There is a need to review the studies with high level of evidence evaluating the effectiveness of nursing interventions performed at the primary care settings.

The objective of this study is to investigate the effectiveness of non-pharmacological community-based nursing interventions for smoking cessation on cessation rate, non-relapsing rate and knowledge, belief and attitude related to smoking in adults living in the community. In this context, this review will aim to address the following questions:

1. Are the non-pharmacological community-based smoking cessation interventions, conducted by nurses in adults living in the community, effective for smoking cessation rate?

2. Are the non-pharmacological community-based smoking cessation interventions, conducted by nurses in adults living in the community, effective to non-relapse?

3. Are the non-pharmacological community-based smoking cessation interventions, conducted by nurses in adults living in the community, effective to change knowledge, belief and attitudes related to smoking positively?

4. Depending on the type of the smoking cessation interventions, is there any difference in quitting smoking rate, non-relapse rate, knowledge belief and attitude changes related to smoking?

5. According to the implication time of the smoking cessation interventions, is there any difference in quitting smoking rate, non-relapse rate, knowledge belief and attitude changes related to smoking? 


\section{METHODS}

\subsection{Study design and population}

Our study will investigate the effectiveness of non-pharmacological community-based nursing interventions for smoking cessation in adults living in the community in this systematic review. This protocol was prepared according to PRISMA-P guidelines (Shamseer et al., 2015).

\subsection{Types of interventions}

The effectiveness of non-pharmacological smoking cessation interventions performed by nurses in adults as individually or group in the context of preventive health care services will be assessed.

\subsection{Types of outcomes}

"Smoking cessation rate" and "non-relapse rate" are the primary outcomes will be evaluated. Secondary outcomes are "changes in knowledge, belief and attitude" of the participants.

\subsection{Types of study to be included}

This review will be comprised of randomized controlled studies and non-randomized studies, including semi-randomized trials, controlled before and after studies and interrupted time series.

Inclusion criteria are as follows:

1. Healthy adults living in the community.

2. Turkish and English studies (research articles, reports and dissertations) published in both refereed and non-refereed journals and electronic journals between January, 2008 and December 2017.

3. Grey literature (specifically Master's and PhD Theses)

4. Studies including community-based non-pharmacological nursing interventions for smoking cessation

5. Studies with appropriate research method (randomized controlled studies, nonrandomized studies)

6. Studies in which the quitting smoking rate was calculated

7. Studies in which the relapses were followed-up

8. Studies in which the duration of smoking cessation interventions carried out by nurses were identified

9. Quantitative studies to be evaluated as "moderate" and "strong" quality via the related quality assessment tool

\subsection{Literature search strategy}

PubMed, Cochrane Library, EMBASE, PsycINFO, Cochrane Central Register of Controlled Trials (CENTRAL), EBSCO, ProQuest, Academic Search Complete, Ovid, CINAHL Plus with Full Text, MEDLINE, Web of Science, Scopus, Turkish Council of Higher Education Thesis Center, Ulakbim Turkish Medical Database and Turkiye Citation Index were searched. Databases were searched from the 1st of January 2008 to the 31st of December 2017. Languages were Turkish and English. 
The following search terms and keywords were used: nicotine (cigarette, tobacco), smoking cessation (quit smoking, tobacco cessation), program (strategy, intervention), health promotion, nursing intervention/s, non-pharmacological interventions, middle aged, adult, community health nursing, tobacco control, randomized controlled study, controlled clinical trials, non-randomized study etc. and different combinations of their Turkish synonyms. MeSH (Medical Subject Headings) terms were taken into consideration in the selection of keywords. The literature searches were conducted as a three-stage approach. Firstly; all the words which were in the titles, abstracts and keywords of the studies, associated with the research question, with the initial keywords were identified. Secondly; included databases were searched in depth via the all identified keywords and terms with various combinations. This stage was conducted under the instructions and counselling of the expert librarians. Thirdly; the reference lists of the identified studies were searched for the purpose of identifying the new studies which were not found in the first and second searching stages. Manuel searches were performed in every stage of the searching process.

\subsection{Data extraction}

Initially, the studies obtained by screening will be listed according to the title, abstract and first author's name by two researchers from the review group. Secondly, the listed studies which meet the inclusion criteria will be specified by the two independent researchers who have worked on the related research topic. Lastly, the full texts of the eligible studies will be downloaded after the duplicated ones are discarded. The EndNote-X8 program will be used to check the duplications.

All the included studies will be noted down on a code table which consists of the aim, study design, sample size, intervention and control groups, type of the intervention, type of the control, duration of the intervention, statistical methods, effect size, findings and quality of the studies.

\subsection{Risk of bias (quality) assessment}

Quality assessment (risk of bias assessment) will be conducted by two independent reviewers for each eligible study via The Quality Assessment Tool for Quantitative Studies (QATQS).

The QATQS was developed in the context of Effective Public Health Practice Project in Canada.(Thomas, Ciliska, Dobbins, \& Micucci, 2004) The tool assesses the quality of different designs of quantitative public health studies, related to family health, sexual health, and prevention from chronic diseases, injuries, and substance use. It consists of eight parts, which are; "bias of selection", "study design", "confounder", "blinding", "data collection method", "exclusion from and withdrawal from the study", "integrity of intervention" and "analysis". Each part, except for the last two ("integrity of intervention" and "analysis"), is scored three Likert type as strong (1), moderate (2), and poor (3). Once all the parts have been scored, a general scoring is performed based on the QATQS assessment dictionary. A methodologically strong study has zero "poor" score, a moderately reliable study has one "poor" score and a methodologically unreliable study has two or more "poor" scores in general assessment. It was validated into Turkish by Ergin and Akın (2018). The content validity was found as .99.(Ergin \& Akın, 2018)

\subsection{Strategy for the data synthesis}

The studies assessed and agreed to be included in this review will be grouped on a standardized form which composed of study design, sample size, interventions and outcomes. The missing information will be requested from the authors of the selected studies. 


\subsection{Ethical Approval}

Before the data collection, ethics committee approval was obtained from Ethics Commission of the university (10.10.2017-08).

\section{RESULTS}

The study is ongoing. The databases were searched and 796 studies were retrieved in total (Academic Search Complete-Ulakbim-CINAHL+with full text:6, CENTRAL:299, OVID/EBSCO-MEDLINE:30, PROQUEST:50, PSYCINFO:3, PUBMED:259, SCOPUSEMBASE:128, WoS:21, the rest had zero study). The quality assessment process was completed. Data extraction is ongoing.

\section{DISCUSSION AND CONCLUSION}

The effectiveness of non-pharmacological smoking cessation interventions performed by nurses in the primary care settings will be assessed in light with the parameters stated above in this systematic review. The data gathered from this systematic review is predicted to be a source for both primary care nurses and the other primary care professionals in terms of the effective smoking cessation method, its duration and changes in knowledge belief and attitude of individuals. Knowledge and recommendations to the literature associated with the research question will be presented via this study. Furthermore, it is aimed to contribute to nursing education curriculum by revealing the roles and influence of primary care nurses on smoking cessation promotion.

\section{Acknowledgement}

This is the protocol of a PhD. thesis with the same topic, which was registered to the International Prospective Register of Systematic Reviews (PROSPERO) on 07.02.2018. PROSPERO 2018 CRD42018088007. Available from: http://www.crd.york.ac.uk/PROSPERO/display_record.php?ID=CRD42018088007. We would like to thank all the members of the thesis jury for their time, support and effort.

\section{Conflict of interest}

No conflict of interest has been declared by the authors. 


\section{REFERENCES}

1. WHO. Global action plan for the prevention and control of non-communicable diseases 2013-2020. WHO Library Cataloguing-In-Publication Data. 2013:1-52.

2. Jamal A, Phillips E, Gentzke AS, et al. Current Cigarette Smoking Among Adults - United States, 2016. MMWR Morbidity and mortality weekly report. 2018;67(2):53-59. doi: https://doi.org/10.15585/mmwr.mm6702a1

3. WHO. WHO global report on trends in prevalence of tobacco smoking 2000-2025. 2nd ed. Geneva: World Health Organization; 2018.

4.TUIK. Türkiye Sağlık Araştırması-2016. 2017; http://www.tuik.gov.tr/PreHaberBultenleri.do?id=24573. Accessed 4th June, 2017.

5. Asma S, Mackay J, Song S, et al. The GATS Atlas. Atlanta, GA: CDC Foundation; 2015.

6.TCSaglikBakanligi. Ulusal Tütün Kontrolü Eylem Planı 2015-2018. 2015; http://www.havanikoru.org.tr/dosya/eylem_plani/ulusal-tutun-kontrol-programieylem-plani.pdf. Accessed 4th July, 2017.

7.Chen D, Wu LT. Smoking cessation interventions for adults aged 50 or older: A systematic review and meta-analysis. Drug and Alcohol Dependence. 2015;154:14-24. doi:https://10.1016/j.drugalcdep.2015.06.004

8.Byers MA, Wright P, Tilford JM, Nemeth LS, Matthews E, Mitchell A. Comparing Smoking Cessation Outcomes in Nurse-Led and Physician-Led Primary Care Visits. Journal of Nursing Care Quality. 2018;33(3):272-278. doi:https://10.1097/NCQ.0000000000000296

9.Hughes JR, Naud S. Perceived role of motivation and self-efficacy in smoking cessation: A secondary data analysis. Addictive Behaviors. 2016;61:58-61. doi:https://doi.org/10.1016/j.addbeh.2016.05.010

10.Moura M, Menezes M, Mariano R, Silva V, Sousa L. Nursing interventions on tobacco control: An integrative review. Revista Brasileira de Cancerologia. 2011;57(3):411419.

11.Elshatarat RA, Yacoub MI, Khraim FM, Saleh ZT, Afaneh TR. Self-efficacy in treating tobacco use: A review article. Proceedings of Singapore Healthcare. 2016;25(4):243248. doi:https://doi.org/10.1177/2010105816667137

12.Nichter M, Carkoglu A, Nichter M, Ozcan S, Uysal MA. Engaging nurses in smoking cessation: Challenges and opportunities in Turkey. Health Policy. 2018;122(2):192197. doi:https://10.1016/j.healthpol.2017.12.007

13.Sarna L, Bialous SA, Chan SS, Hollen P, O'Connell KA. Making a difference: Nursing scholarship and leadership in tobacco control. Nursing Outlook. 2013;61(1):31-42. doi:https://doi.org/10.1016/j.outlook.2012.05.007

14.Rice VH, Hartmann-Boyce J, Stead LF. Nursing interventions for smoking cessation. The Cochrane database of systematic reviews. 2013(8):Cd001188. doi:https://10.1002/14651858.CD001188.pub4

15.Rice VH, Heath L, Livingstone-Banks J, Hartmann-Boyce J. Nursing interventions for smoking cessation. The Cochrane database of systematic reviews. 2017;12:Cd001188. doi:https://10.1002/14651858.CD001188.pub5 
16.Zbikowski SM, Magnusson B, Pockey JR, Tindle HA, Weaver KE. A review of smoking cessation interventions for smokers aged 50 and older. Maturitas. 2012;71(2):131-141. doi:https://10.1016/j.maturitas.2011.11.019

17. Shamseer L, Moher D, Clarke M, et al. Preferred reporting items for systematic review and meta-analysis protocols (PRISMA-P) 2015: elaboration and explanation. BMJ. 2015;349:g7647. doi:https://doi.org/10.1136/bmj.g7647

18.Thomas BH, Ciliska D, Dobbins M, Micucci S. A process for systematically reviewing the literature: providing the research evidence for public health nursing interventions. Worldviews on evidence-based nursing. 2004;1(3):176-184. doi:https://doi.org/10.1111/j.1524-475X.2004.04006.x

19.Ergin E, Akın B. The Turkish adaptation of a quality assessment tool for quantitative studies: validity and reliability analyses. Turkiye Klinikleri Journal of Nursing Sciences. 2018;10(4):292-308. doi:https://10.5336/nurses.2018-61334 